\title{
Investigation \& Scrutiny of Protected Assignment Supervision Systems for Mobile Enabled with Internet Protocol Version 6 Networks
}

\author{
Arpit Bhushan Sharma ${ }^{1, *}$, Harshit Kargeti \\ ${ }^{1}$ Department of Electrical \& Electronics Engineering, KIET Group of Institutions, Ghaziabad, Uttar Pradesh, India \\ ${ }^{2}$ Department of Information \& Technology, ABES Institute of Technology, Ghaziabad, Uttar Pradesh, India \\ Email address: \\ arpit3043@gmail.com (A. B. Sharma), hkargeti@gmail.com (H. Kargeti) \\ ${ }^{*}$ Corresponding author \\ To cite this article: \\ Arpit Bhushan Sharma, Harshit Kargeti. Investigation \& Scrutiny of Protected Assignment Supervision Systems for Mobile Enabled with \\ Internet Protocol Version 6 Networks. American Journal of Computer Science and Technology. Vol. 3, No. 4, 2020, pp. $76-85$. \\ doi: $10.11648 /$ j.ajcst.20200304.12
}

Received: September 28, 2020; Accepted: November 2, 2020; Published: November 11, 2020

\begin{abstract}
Advancement in remote technologies advances the grants of Internet Protocol version 6 (IPv6) empowered handheld cell phones to get to the cyberspace globally. An Internet Protocol address is requisite to perceive the internet versatile cloud-host system. Compact (Mobile Internet Protocol version 6) MIPv6 scheme engages a flexible host to move beginning with one subnet then onto the following without changing its leaving Internet Protocol address. For IPv6 based adaptability, IETF proposed that Standard Mobile IPv6 as first movability the officials scheme. It gives an incredible and adaptable approach to deal with handover the executives. Standard Mobile Internet Protocol version 6 is a host-based worldwide versatility the board plot and suffer from essential issues, for example, flagging overhead, handover idleness and parcel misfortune. Accordingly, IETF has normalized confined system based Versatility Management intends to vanquish issues related with overall host-based convey ability the board plans termed as Proxy Mobile Internet Protocol version 6 (PMIPv6). It decreases handover inactivity and parcel misfortune contrasted with have based versatility the executives conspire impressively, yet, experiences security issues. Later on, scientists planned made sure about PMIPv6 conventions for confirmation of portable just as system gadgets inside LMD. The paper audits different handover the board plans for secure handover the executives. The exhibition of different plans subjectively examined on fundamental boundaries, for example, verification cost, flagging cost, parcel misfortune and so forth.
\end{abstract}

Keywords: HMIPv6, Handover Management, MIPv6, PMIPv6, Packet Loss, S-PMIPv6

\section{Introduction}

Headway in remote and versatile advancements permits handheld cell phones to get to the Internet offices universally [1]. In the ebb and flow situation, the versatile administrators are searching for the cutting edge system to offer consistent support in a productive and savvy way. Fruitful execution of bundle based portable systems [2, 3] have pulled in versatile administrators. These systems have capacity to deal with an enormous number of supporters and stream of traffic capacity. In bundle created administrations, all information parcels are autonomous to each entrance to the systems. Figure 1 indicates the expectation from CISCO on versatile information traffic development. In the most recent decade, numerous building arrangements have been recommended for consistent administrations to the clients of Internet. Worldwide Interoperability for Microwave Access (WiMAX) [5] and (EPS) [4] Evolved Packet System are most appropriate instances of these models utilized for mutually speech and information administrations

Versatility for the board which is the essential matter for the Succeeding for Generation of $\mathrm{WiFi}$ and a chain of interconnected Mobiles. The Internet Protocol based versatility the executive's conventions assume huge job in meeting congruity, once a node of cellular phone travels over the systems. The fundamental goal of portable IP is to give a continuous correspondence administration. It empowers the allocation systems to find the nodes of cellular phone (MN) 
which present the Point of contact (PoC) to the cyberspace. These aides in conveying information parcels to the node of cellular phone alongside keeping up association with nodes of cellular phone (MN) as it changes its $\mathrm{PoC}$ ceaselessly. The way toward distinguishing the $\mathrm{PoC}$ in allocation system to convey information bundles is recognizing as area the board [6]. Then again, the way toward keeping up the supporter association with least corruption in Quality of Service (QoS) [7], for example parcel misfortune, start to finish delay and so on., is recognize as handover the board $[8,9]$. The $\mathrm{MN}$ encounters the handover at whatever point it moves starting with one system then onto the next. It is a tedious procedure and during this time, MN can't impart from Correspondent Node (CN) [10]. The deferral brought about throughout assignment is recognize as handover inertness. [11, 12]. Reducing the handover delay is one of key issues of MIPv6 technology. The FMIPv6 mechanism reduces address configuration delay to speed handover process; the HMIPv6 mechanism uses MAP management to reduce the binding update delay and network signaling load. The FHMIPv6 mechanism is more suitable for the multimedia real-time requirement, but it increases the MAP's load dealing with signaling interactions. This paper also proposes several optimization programs about the delay of DAD and BU, but these programs just past simulation, and still need to be validated and improved in real network environment. [13]. Particularly when placed in the backbone network, the result of IPv6 traffic control module is very good, and has good scalability. Net filter framework has good flexibility and can be easily extended. To the emerging protocols it can be implemented new rules and module, thus we can design a cheap, safe, practical and flexible traffic control system for IPv6 [14]. Mobility without changing the IP address is the key to the mobile IPv6. The key technology for mobile IPv6 is the mobile switching process. How to reduce the switching delay is the standard to measure the function of the mobile IPv6 switch and for this reason, as for the mobile host's frequent switching in the field network we propose the Hierarchical Mobile IPv6 to reduce the delay, and expect the switch of the second layer's trigger has made rapid switching for mobile IPv6 [15]. In this paper, we propose an improved program based on Investigation \& Scrutiny of Protected Assignment Supervision Systems for Mobile Enabled with Internet Protocol Version 6 Networks and subsidiary by different procedural aspects and different Protocols.

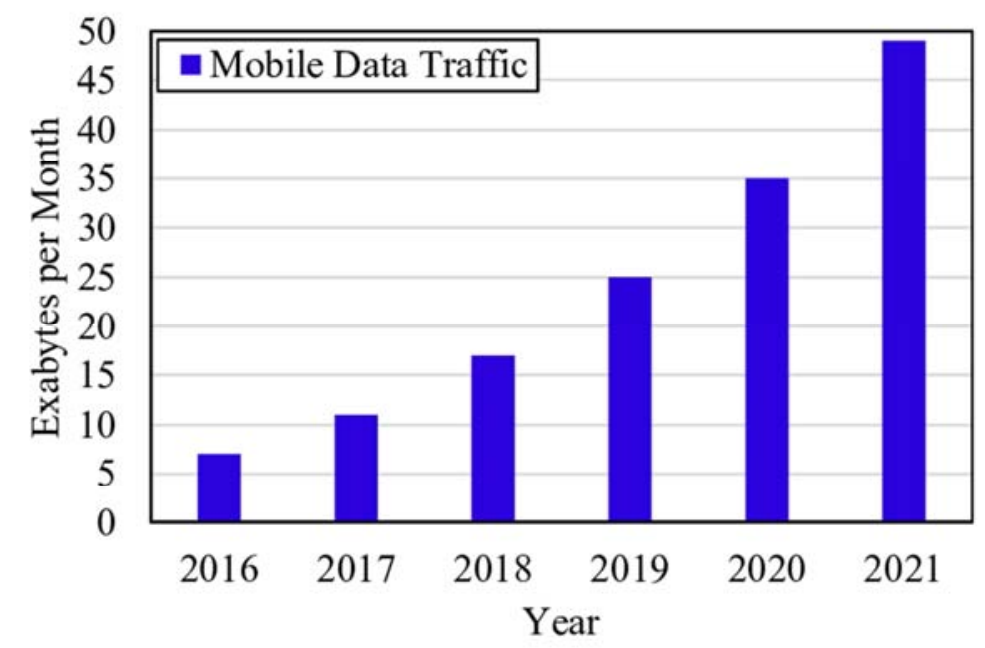

Figure 1. CISCO expectation on versatile information traffic development for 2016-2021.

\section{Related Work}

The $\mathrm{MN}$ is in every case extraordinarily distinguish by IP-address, distributed by home system for all time to MN stays inside the limit of the system. During development, when the MN relocates from the limit of existing system, continuous meeting promptly ends and to continue Cyberspace network it demands another Internet Protocol address. An allocation system relegates to the $\mathrm{MN}$, another Internet Protocol address for continuing the administrations. MIP was proposed by the Internet Engineering Task Force (IETF) to give continuous portability to MN. MIP underpins the development of the nodes of cellular phone (MN) without evolving Internet Protocol address ending the meeting. IETF proposed First versatile IP was variant (MIPv4) [9] 4. It experiences a few basic issues, for example, constrained location space, less security issue, triangular directing and so forth. To defeat from issues connected with MIPv4, the IETF has recommended versatility the board conventions dependent on Internet Protocol form-six. The Conventions are order into bi-gatherings: Cloud based portability the board plans and Network-based versatility the executives plan [10].

1. Mobility Management Protocols (Host-based)

In host based versatility the executives' conventions, portable host is responsible to completing portability related flagging. Portable (MIPv6) [11] IPv6 is primary host based versatility the executives' convention recommended by IETF for IPv6. In this way, IETF put forward improved forms, for example, FM-Internet Protocol version 6, HM- Internet Protocol version $6 \&$ so forth. 


\section{Mobile Internet Protocol version 6}

It is main worldwide versatility the board convention. It gives reachability and meeting congruity for the nodes of cellular phone $(\mathrm{MN})$, while circling the IPv6 systems. In Mobile IPv6, nodes of cellular phone (MN) is answerable for all versatility related flagging. Every nodes of cellular phone $(\mathrm{MN})$ is remarkably recognized by an extraordinary Internet Protocol version 6 address, also known as Home Address (HoA), regardless of Point of Contact to the Home Network Cyberspace. A hub that speaks to the $\mathrm{MN}$ is called a Correspondent Node. Mobile Internet Protocol version 6 presents an uncommon system substance called as Home Agent. The HA is answerable for continuous correspondence administrations among $\mathrm{CN}$ and $\mathrm{MN}$.

HA is executed in a home system through an extraordinary switch. The HA fills in as a passage for the system and is answerable for dealing with all parcels leaving or entering the system. At the point When the nodes of cellular phone (MN) visits other system outside the $\mathrm{HN}$, the HA blocks all parcels coordinated to the nodes of cellular phone (MN) via the $\mathrm{CN}$ and searches for the CoA and HoA pairs in the Binding Cache Entry. Having regard to the enlisted CoA, the HA exemplifies the bundles obtained and passes them to the present area of MN. Thus, IPv6 parcels sent by nodes of cellular phone (MN) are primarily burrowed at HA and then steered to their last target, for example $\mathrm{CN}$. This methodology shapes a triangular steering, resulting in a wasteful use of transfer speed. Figure 2 and Figure 3 show parcel conveyance flow in Mobile Internet Protocol version 6, when nodes of cellular phone (MN) is visiting another system individually and when the nodes of cellular phone (MN) is in its $\mathrm{HN}$.

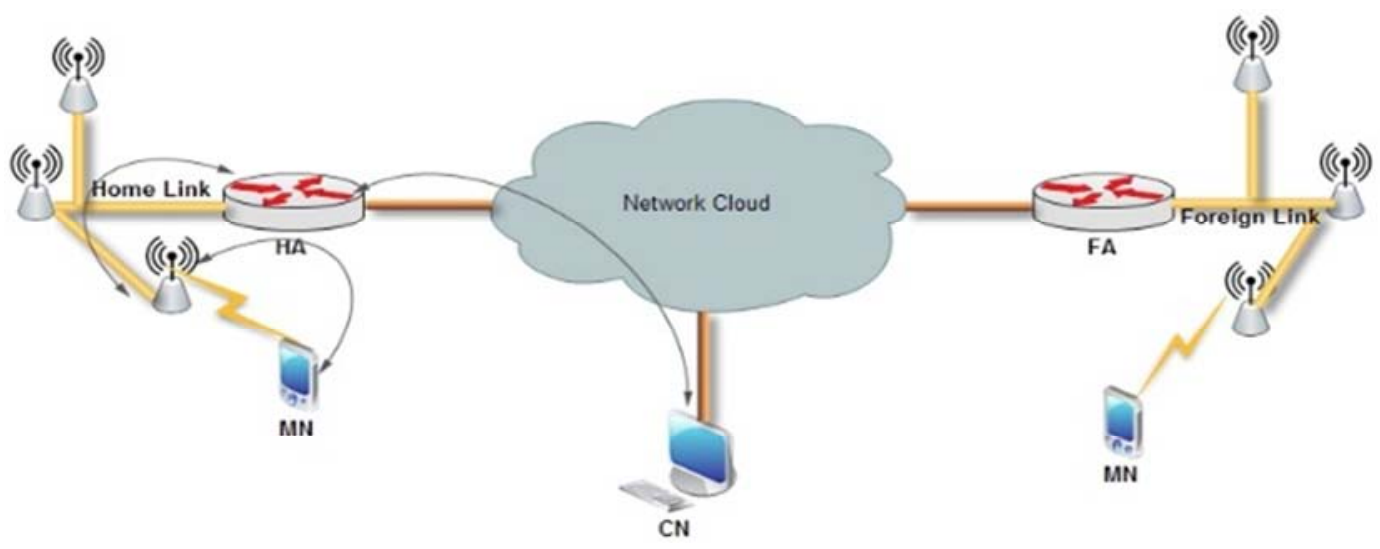

Figure 2. Container transfer process when MN is in home network in MIPv6.

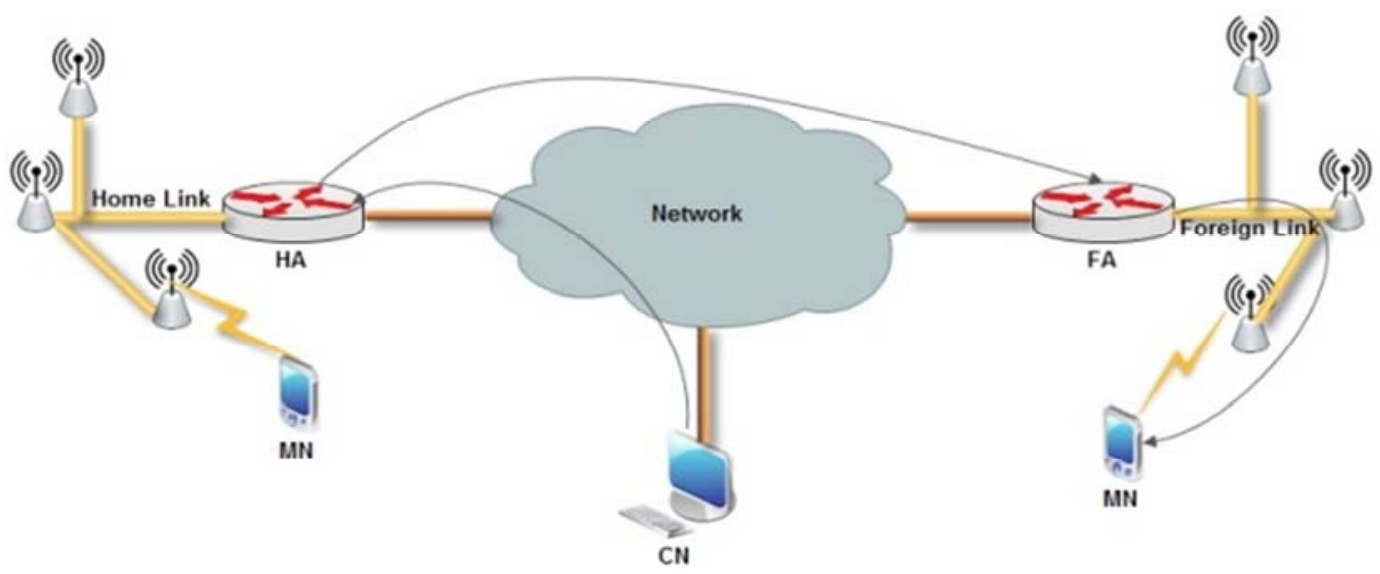

Figure 3. Container transfer process when nodes of cellular phone (MN) in visiting network in MIPv6.

Following are the signaling steps in MIPv6:

Stage 1: - In a meeting system, MN shoots Router Solicitation (RS) communication to find the nearness to Internet Protocol version 6 switches on the connection.

Stage 2: - Because of Router Solicitation, the switches on connect answer by shooting Router Announcement communication having a rundown to precedes maybe be used for IP design.

Stage 3: - Other IP-address is arranged by the MN, also known as (TCoA) Tentative Care-of Address. MN communicates (NS) Network Solicitation message to every single neighboring hub to confirm the uniqueness of Tentative Care-of Address.

Stage 4: - If some other gadget on the system utilizing identical IP address reacts quickly to $\mathrm{MN}$ by shooting (NA) Network Advertisement message. The way toward confirming the unlikeness of Tentative CoA is identified as (DAD) Duplicate Address Detection. On the off chance that MN doesn't get any reaction inside the time apportioned by arrange, the Tentative Care-of Address is allocated as the CoA to the 
nodes of cellular phone $(\mathrm{MN})$.

Stage 5-: The nodes of cellular phone (MN) illuminates by shooting the (BU) Binding Update message with CoA about the present area at the HA. The HA retains on the BCE the official of the MN between the CoA and the HoA. On every IP handover $\mathrm{MN}$ refreshes the coupling itself. With the new CoA, the BCE, The HA refreshes

Stage 6-: The HA educates about fruitful enlistment by shooting Binding Acknowledgment message towards the MN. The flagging arrangement outline speaks to the real progression of information bundles. Figure 4 shows flagging grouping outline for MIPv6:

Uses the Return Rout Ability Procedure to mitigate the Mobile Internet Protocol version 6 presentation. It allows for direct correlation between $\mathrm{CN}$ and nodes of cellular phone (MN). Registry Registrar Protocol (RRP) is liable for making sure that challenge-reaction messages improve course with the help. RRP- Registry Registrar Protocol, 4- messages: Homebased Test Inuit, Home Test, Test Care Init, and Test Caution are trade between the nodes of cellular phone (MN) and the $\mathrm{CN}$ to check the $\mathrm{CN}$ 's personality. MN legitimately sends a HoTI response to $\mathrm{CN}$ with HA compromising 64-piece Nonce then CoTI communication to the $\mathrm{CN}$ comprising exactly the similar Norms. $\mathrm{CN}$ responds to the nodes of cellular phone (MN) by sending HA a HoT message with Nonce sent by MN along with 64 pieces Nonce produced by $\mathrm{CoT}$ and $\mathrm{CN}$ message having Nonce sent by $\mathrm{MN}$ along with 64 pieces Nonce created by $\mathrm{CN}$. With the help of the communications nodes of cellular phone (MN) confirms CN's respectability. $\mathrm{CN}$ can then speak with $\mathrm{MN}$ straightforwardly after fruitful check. Because of enhancement of course, the issue of bottleneck at HA diminishes substantially alongside better use of transmission capacity. Figure 5 displays the immediate transmission of the bundle after the nodes of cellular phone $(\mathrm{MN})$ visits another system.

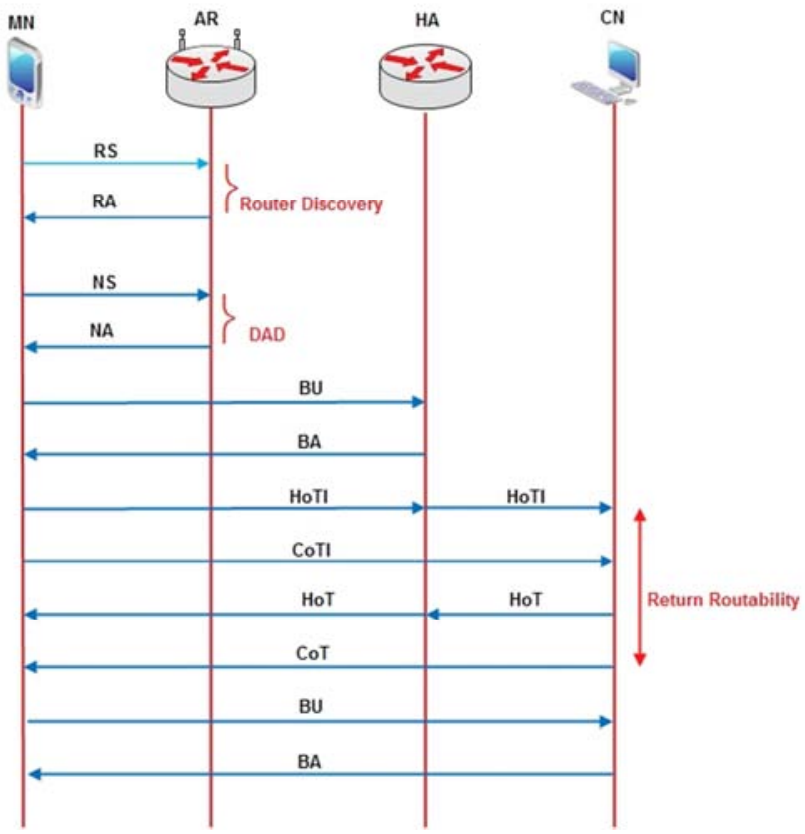

Figure 4. MIPv6 diagram.

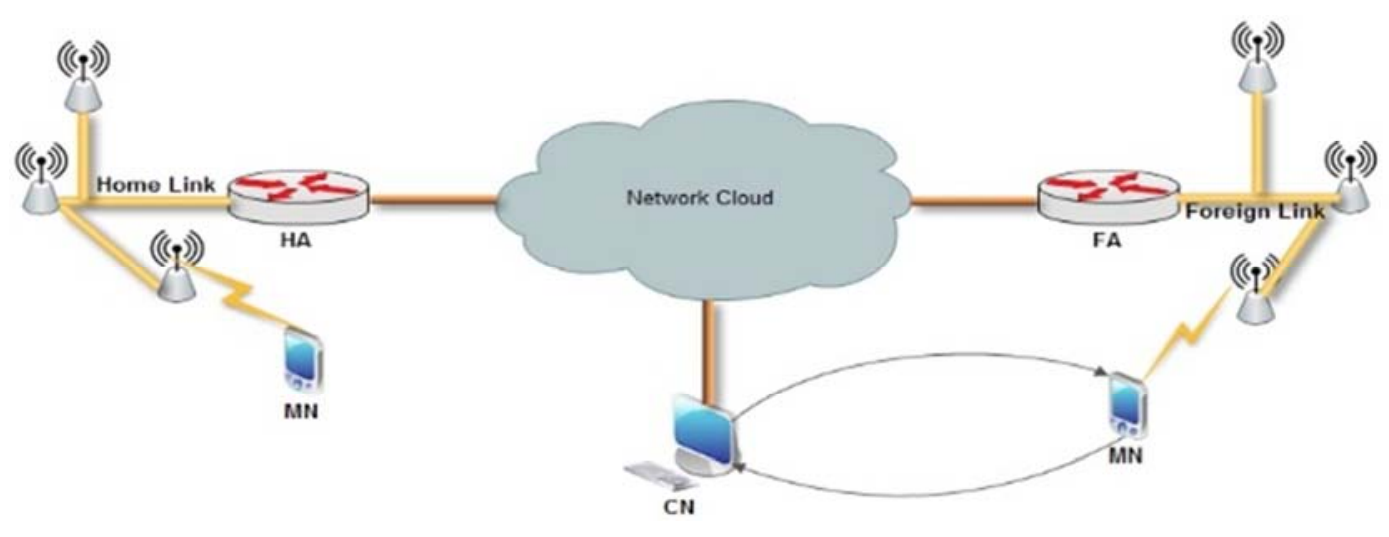

Figure 5. CN to $M N$ (Delivering packets directly).

\section{Hierarchical Mobile IPv6}

Hierarchical Mobile IPv6 has been put forth as an augmentation to the Mobile Internet Protocol version 6 convention. HMIPv6 gives both smaller scale versatility just as large scale portability. HMIPv6 presents another element, example, Mobility Anchor Point (MAP). The Mobility Anchor Point is like the Home Agent Inside Mobile IPv6 with additional highlights. MAP isn't limited to be set at the limit of the system, it very well may be put anyplace inside the switches chain of command. The MAP groups the system in sub-domains and limits flagging overhead altogether by outside spaces. This procedure mitigates handover effectiveness of system. At the point in the MAP area when a $\mathrm{MN}$ enters first time, it needs to educate its new area to the $\mathrm{CN}$ and HA with the assistance by BU messages just a single time. From that point forward, the MN moves unreservedly inside the MAP space without educating CN or HA. Figure 6 depicts the design and bundle conveyance component for Hierarchical Mobile Internet Protocol version 6. In HMIPv6 systems, the $\mathrm{MN}$ is distinguishing by 2 locations in particular, Regional Care-of Address and On-Link Care-of Address. 


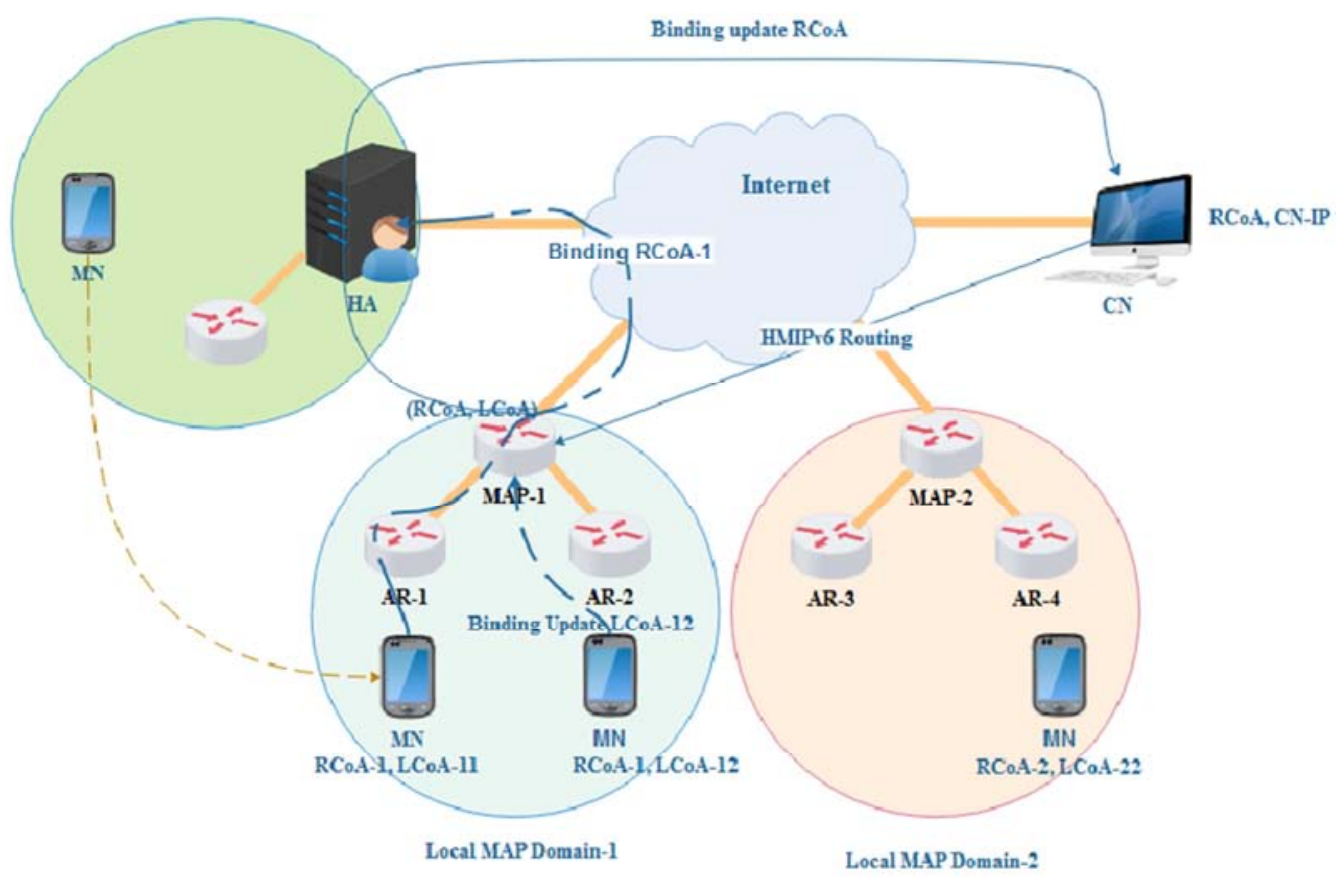

Figure 6. HMIPv6 planning.

LCoA is considered to be the CoA cast-off through MAP, besides RCoA is considered to be the CoA cast-off by HA \& $\mathrm{CN}$ following advancement of the course. The MAP preserves BCE section relating to separate pair of an LCoA and an $\mathrm{RCoA}$. When RCoA is relegated to the nodes of cellular phone $(\mathrm{MN})$ the situation remnants stable till nodes of cellular phone (MN) passes its MAP limit. The nodes of cellular phone (MN) promptly shoots a Local Necessary Update communication to MAP in case the MN is associated with the new access switch in the wake of leaving the past one and MAP quickly refreshes BCE table to a new LCoA. MAP advises MN by shooting Local Binding Acknowledgment about fruitful enlistment at the MAP. The MAP limit in the wake of intersection, for example, the MN needs to educate its current area PoA to $\mathrm{CN}$ and HA. Due to limited portability, the overhead flagging in Hierarchical Mobile Internet Protocol version 6 decreases completely, resulting in extensive improvement in idleness and parcel misfortune.

Following are the signaling steps in HMIPv6:

Stage 1-: The nodes of cellular phone $(\mathrm{MN})$ directs RS communication to find the nearness of Internet Protocol version 6 switches in the MAP space.

Stage 2-: The switches inside MAP space react to MN by distribution of a message to Router Announcement (RA) comprising a slope of precedes that can be used to set up the address.

Stage 3-: The nodes of cellular phone (MN) arranges another version 6 address of the Internet Protocol, recognized as Tentative Care-of Address (TCoA), with the help of precedes obtained from ARs. MN communicates Network Solicitation (NS) message to every neighboring hub to confirm the uniqueness of TCoA.

Stage 4-: By sending Network Advertisement (NA) message, if some other gadget in the system using the same IP address reacts quickly to nodes of cellular phone (MN). Duplicate Address Detection (DAD) [12-15] is supposed to be the way to checking the uniqueness of TCoA. If nodes of cellular phone $(\mathrm{MN})$ does not receive any reaction within the time specified by the organization, the TCoA currently allocated to $\mathrm{MN}$ as LCoA.

Stage 5-: Cell phone nodes (MN) advise the MAP about the new LCoA by shooting the message Local Binding Update. MAP maintains the MN's official position in BCE between LCoA and RCoA.

Stage 6-: MAP also illuminates the enrollment effectively by shooting the LBA communication to $\mathrm{MN}$.

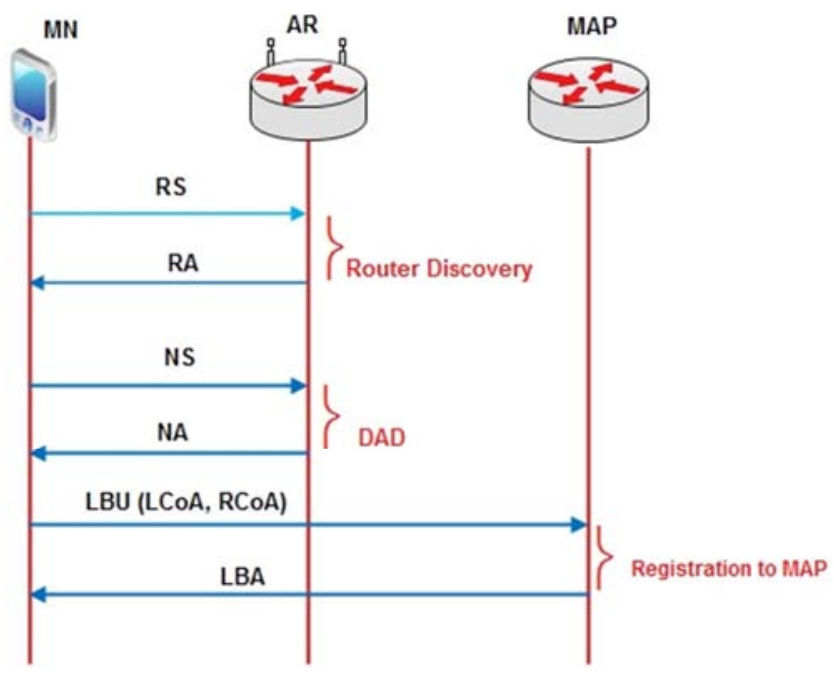

Figure 7. HMIPv6 Signaling order diagram.

2. Mobility Management Protocols (Network-based)

In arrange based portability the board convention, the system elements are mindful to carryout versatility related flagging. The primary system based versatility the board 
convention proposed for Internet Protocol version 6 systems by IFTE was PMIPv6. Following that, scientists put forth improved renditions of PMIPv6, regarded as Securde-PMIPv6. System based versatility the executive's conventions give better exhibitions over host-based portability the board conventions, yet at the same time experience the ill effects of flagging overhead handover inactivity, security issues, and parcel misfortune issues while handover.

3. Secured-Proxy Mobile Internet Protocol version 6

Secured Proxy MIPv6 is an enhanced IPv6 portability convention that utilizes the idea of MIPv6 and ameliorates the versatility the executives in Restricted Mobility Domain. The Secured Proxy Mobile IPv6, nodes of cellular phone (MN) doesn't need any kind of unique programming inform to give IP-based portability provision. Every portability related signs are complete by arrange substances. The substances partaking in versatility the board are Local Mobility Anchor, Authentication Authorization Accounting server and Mobile Access Gateway. The MAG functions as Access Router and deals with the versatility related motioning for the benefit of nodes of cellular phone (MN).
MAG is situated among LMA and nodes of cellular phone $(\mathrm{MN})$. The fundamental obligations of MAG are; following the developments of MN inside the LMD, starting the versatility related motioning with LMA for the benefit of the MN and setting up burrow among MAG and LMA. Then again, LMA consumes identical obligations as HA in the MIPv6. It is additionally recognizing as the door to the LMD. LMA keeps up the BCE best ever for every MNs and MAG related with it. Therefore, Internet Protocol version 6 parcels coordinated to the MN from $\mathrm{CN}$ are sent to LMA first. LMA captures the all bundles and passages them after epitome to Proxy Care-of Address for example address of MAG. The MAG encapsulates got parcels and conveys them to MN. So also, Internet Protocol version 6 bundles sent by $\mathrm{MN}$ are exemplified at MAG, burrowed to LMA, and afterward directed to its last goal for example $\mathrm{CN}$. In made sure about PMIPv6, Authorization, Authentication, Accounting server is answerable for keeping up profile of every $\mathrm{MN}$ and $\mathrm{MAG}$ inside the domain of mobility limitations. AAA essential validate all gadgets previously speaking with the CN. Figure 8 depicts engineering for made sure about Proxy Mobile IPv6 plot.

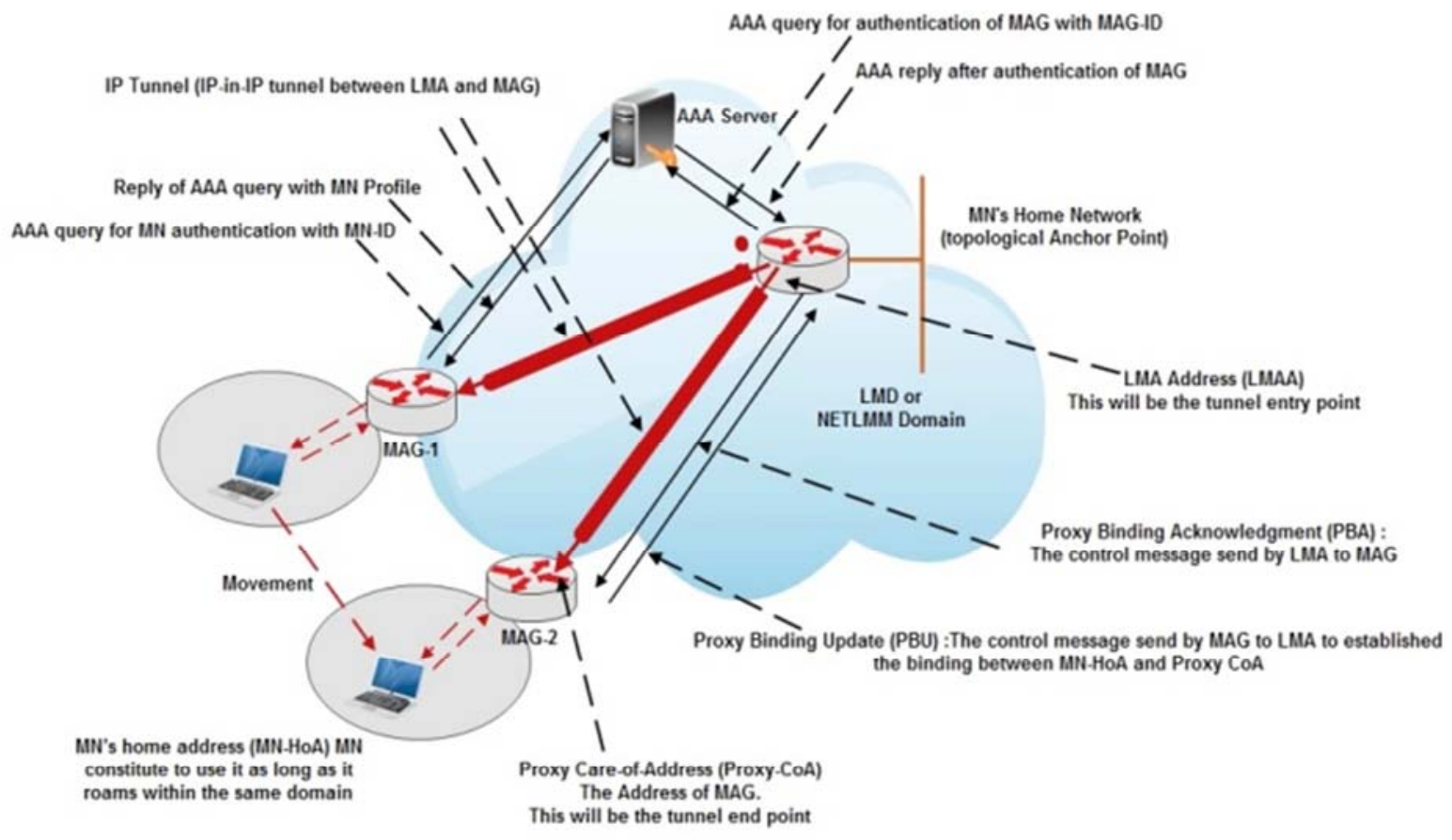

LMA: Local Mobility Anchor

MAG: Mobile Access Gateway

AAA: Authentication, Authorization and Accounting

LMD: Local Mobility Domain

METLMM Domain : Network-based Localized Mobility Management Domain

Figure 8. Architecture for Secured-Proxy Mobile Internet Protocol version 6.

Following are the signaling steps in S-PMIPv6:

Stage 1-: When MN shows up at another Localized Mobility Domain (LMD) in the wake of leaving the past one or switches on power in existing LMD, the MAG promptly identifies the nearness of MN.

Stage 2-: Subsequently recognizing the nodes of cellular phone (MN) connection, MAG gets answerable for confirmation of the MN's character. For confirmation, the
MAG solicitations to the AAA server by sending the MN-Identifier (MN-ID) and MAG-Identifier (MAG-ID). The AAA server confirms the MN-ID from a current database. On the off chance that the $\mathrm{MN}$ is validated effectively, AAA server reacts to MAG by sending MN's and MAG's profile, comprising of subnet address, the MN's home system address and the other needed data demanded to enroll at the LMA.

Stage 3-: MAG demands LMA enrollment of the MN by 
shooting PBU message. PBU incorporates MN-ID, the Proxy-Care of Address, MAG-ID and the coupling generation of the MN's and so forth.

Stage 4-: After getting PBU, LMA directs the AAA-question communication to A-A-A-server for check of the credibility of PBU correspondent.

Stage 5-: The AAA attendant servers scans for the MAG-ID in its database for check and on effective confirmation, it quickly reacts to LMA by AAA response message.

Stage 6-: The fruitful confirmation from the AAA attendant servers, LMA scans for MN's entrance in the BCE. On the off chance that passage exists, LMA promptly refreshes MN's connection to the new MAG-ID or the LMA quickly makes new record for the $\mathrm{MN}$ in the BCE. The LMA additionally advises back to the MAG of effective enlistment by shooting a
PBA message. PBA message consists the the Home Network Prefix of MN.

Stage 7-: By accepting PBA, MAG sets up a two directional passage between the MAG and the LMA. This passage is answerable for moving all the information traffic among MAG and LMA.

Stage 8-: MAG educates about effective enrollment at the LMA to the MN by transfer a Router Announcement communication containing the HNP. The usefulness of Authentication Scheme optimized and secured in Proxy Mobile IPv6 plot is like made sure about PMIPv6, with the exception of the verification methodology. During confirmation process, MAG directs A-A-A inquiry message alongside MN-ID and MAG-ID to A-A-A server.

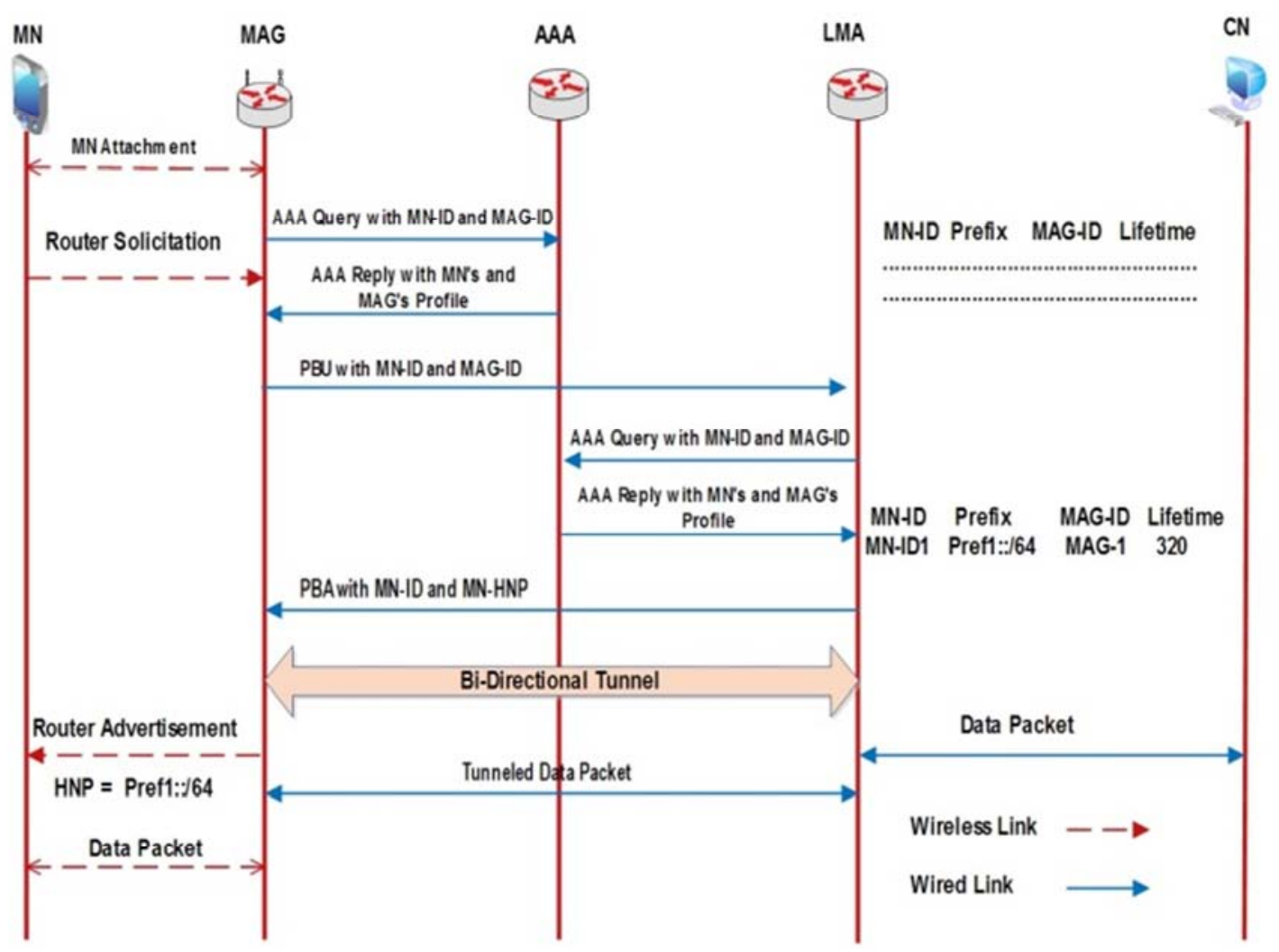

Figure 9. Signaling sequence diagram for Secure- PMIPv6.

The AAA server checks the realness of nodes of cellular phone $(\mathrm{MN})$ and MAG, and shoots multicast message to LMA and MAG. The multiple casting of communicated message contains a MN-profile and MAG-profile required for enrollment at LMA. Figure 4 shows streamlined verification process in SOAS-PMIPv6 by expelling flagging overhead of stage 4 for example by wiping out AAA-inquiry message for same data. Presently, LMA makes another record in BCE for MAG and related MN. By this method one flagging message, (LMA question message to the AAA server for check of MAG's personality) get diminished when contrasted with PMIPv6 and hence diminishes the handover inertness.

4. Secured \& Optomized Authentication Scheme for Proxy Mobile Internet Protocol version 6
Authentication Scheme optimized and secured in Proxy Mobile IPv6 is improved usage of S-PMIPv6. It diminishes excess flagging overhead during confirmation method and it results in a lower flagging expense and parcel misfortune. Figure 10 shows a handover technique in the Secure and Optimized Authentication Scheme in Proxy Mobile Internet Protocol version 6.

Following are the signaling steps in SO-PMIPv6:

Stage 1-: As the MN relocates to a newer LMD from an old LMD or boots up first time in the equivalent LMD, the MAG promptly identifies the nearness of the MN and sets up a highlight point association among $\mathrm{MN}$ and MAG.

Stage 2-: To confirm the validity of the MN, the MAG sends the MN-ID alongside the MAG-ID to A-A-A server. 
Stage 3-: A-A-A server confirms the MN's personality; it shoots a multicast message to MAG and LMA, which contains MN's profile, MAG profile and other data required for enrollment of MN at LMA.

Stage 4-: Over fruitful confirmation, MAG shoots message of PBU to the LMA consists of MN-ID, the MAG-ID and data demanded from the A-A-A server.

Stage 5-: Afterwards fruitful confirmation data from the AAA server, LMA has total data required for enrollment of MAG and related $\mathrm{MN}$ in $\mathrm{BCE}$ at the LMA. It promptly dispenses the Home-Network-Prefix (MN-HNP) and makes another record for MAG and related MN. The BCE includes data, for example, the $\mathrm{MN}-\mathrm{ID}$, the $\mathrm{P}-\mathrm{CoA}$ restricting lifetime, the MN-HNP and so forth.

Step 6-: As the LMA gets PBU note from the MAG alongside effective enrollment in $\mathrm{BCE}$, it promptly reacts to MAG by sending a PBA message containing MN-HNP.

Stage 7-: MAG develops the two dimensional passage between the LMA and the MAG in the wake of getting PBA.
All the bundles from the $\mathrm{CN}$ are divert to $\mathrm{MN}$ through the set up bidirectional passage. The LMA likewise makes a note for MN-HNP in Binding Update List at the MAG.

Stage 8-: MAG likewise illuminates to MN about effective enlistment by shooting the RA letter consisting HNP.

\section{Qualitative Analysis}

This area manages subjectively explore the current made sure about plans in Mobile Internet Protocol version 6 systems dependent on different assessment models, for example, engineering, security, confirmation messages, bundle misfortune and so on and contrast them and existing notable plans. A summation of the fundamental qualities, including the solid and feeble purposes of proposed plans contrasted with the different existing notable Secure and Optimized Authentication Scheme in Proxy Mobile Internet Protocol version 6, HMIPv6, Protocol version 6 of Mobile Internet and S-PMIPv6 plans, is examine in Table 1.

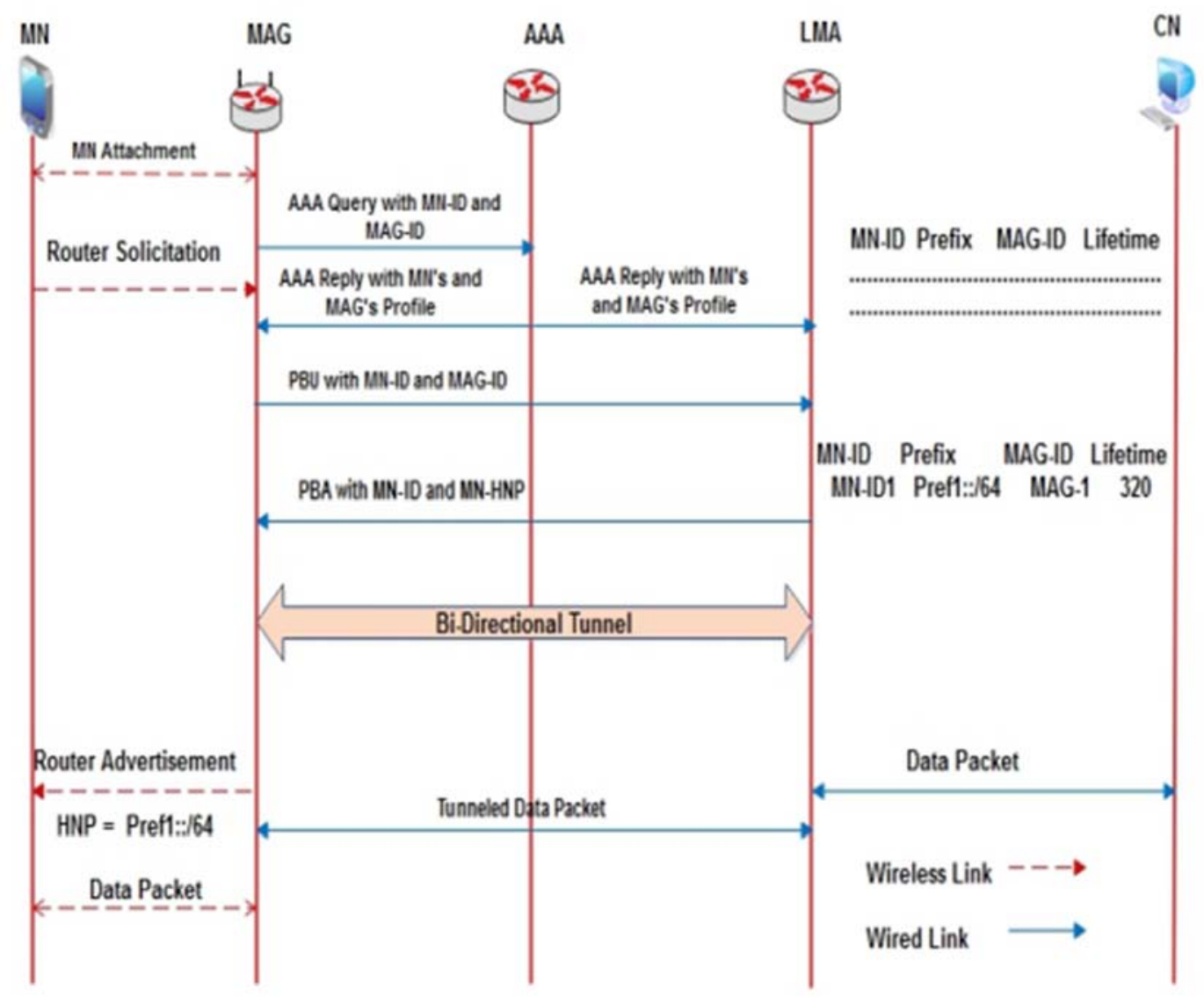

Figure 10. Sending Sequence signal from S-PMIPv6.

In have based versatility the executive's plans, copy address recognition is a tedious procedure and essential driver of bundle misfortune and handover dormancy. Validation delay is another reason for parcel misfortune and handover inertness. During confirmation process, have based plans trade four verification messages between versatile hub and system substances. Rather than have based plans, S-PMIPv6 and Secure and Optimized Authentication Scheme in Proxy
Mobile Internet Protocol version 6 plans give limited system based versatility answer for Internet Protocol version 6 systems. In these plans all flagging related overhead is due by organize substances without cooperation of portable hubs. These plans do exclude copy address location process; bring about lesser handover idleness and parcel misfortune contrasted with have based plans. In light of above conversation, we reason that organize based portability the 
executives' plans are better than have based versatility the executives plan on the grounds that these plans lessen handover inertness (1 second) by evacuating copy address identification process. Battery control is 1 of the basic matter in versatile correspondence. These plans don't include versatile hubs in flagging procedure, in this manner lessening the battery utilization of portable hubs. System based plans don't include portable hubs in verification process, so decreases handling overhead contrasted with have based plans.
The verification flagging overhead additionally assumes a significant job in handover idleness and bundle misfortune. Verification delay relies upon count of messages traded among arrange substances. Optimized and Unassailable Authentication Scheme in Proxy Mobile Internet Protocol version 6 trades less count of messages during confirmation process then existing Sensor Proxy Mobile Internet Protocol version 6, brings about best execution and Quality of Service.

Table 1. (Mobility administration schemes) Qualitative analysis

\begin{tabular}{|c|c|c|c|c|}
\hline Scheme/Aspect & $\begin{array}{l}\text { Mobility Internet } \\
\text { Protocol version } 6\end{array}$ & $\begin{array}{l}\text { Hierarchical Mobile } \\
\text { Internet Protocol } \\
\text { version } 6\end{array}$ & $\begin{array}{l}\text { Secured Proxy } \\
\text { Mobility Internet } \\
\text { Protocol version } 6 \\
\end{array}$ & $\begin{array}{l}\text { Secured \& Optimized } \\
\text { Authentication Scheme for Proxy } \\
\text { Mobile Internet Protocol version } 6\end{array}$ \\
\hline Architecture & Unified & Unified & Unified & Unified \\
\hline Layer of operating & Network Layer & Network Layer & Network Layer & Network Layer \\
\hline Wireless link tunneling & Yes & Yes & No & No \\
\hline Required Infrastructure & $\mathrm{HA}, \mathrm{AR}$ & MAP, AR & MAG, LMA & MAG, LMA \\
\hline Modification in MN's Protocol stack required & Yes & Yes & No & No \\
\hline Optimization of route & Support & Support & Not supported & Supported \\
\hline Address Detection for duplicity & Required & Required & Not Required & Not Required \\
\hline Duplicate Address Detection (Time consumption) & 1 seconds & 1 seconds & Not required & Not required \\
\hline Address (es) of MN & 2 (HoA, CoA) & $2(\mathrm{RCoA}, \mathrm{LCoA})$ & $1(\mathrm{HoA})$ & $1(\mathrm{HoA})$ \\
\hline Method of Data transport & IP tunneling & IP tunneling & IP tunneling & IP tunneling \\
\hline Update at Binding & MN-HA & MN-MAP & MAG-LMA & MAG-LMA \\
\hline Secure Communication & Yes & Yes & Yes & Yes \\
\hline Number of Authentication Messages required & 4 & 4 & 4 & 3 \\
\hline Authentication Server overhead & High & High & High & Least \\
\hline Cost of Signaling & Maximum & Less than MIPv6 & Less than HMIPv6 & Least \\
\hline Latency of Handover & Maximum & Less than MIPv6 & Less than HMIPv6 & Least \\
\hline Loss of Packet & Maximum & Less than MIPv6 & Less than HMIPv6 & Least \\
\hline Service Quality (QoS) & Worst & Better & Better than HMIPv6 & Best \\
\hline
\end{tabular}

\section{Conclusion}

In the following paper, a profound audit of present adaptability the executive's plans are directed. Writing study features amount of solidarity and shortcoming of current host-based and organize based portability the executive's plans. In the most recent decade, huge advancement in field of electronic industry presented top of the line versatile gadgets like PDA, cell phone, tablet and so on in advertise. These gadgets constrained clients to move from a fixed wired system to the remote and portable system. To offer consistent types of assistance to the following gadgets, Task Force of Internet Engineering has presented Mobile IPv4 as host-based portability the board convention. It can offer relentless types of assistance to portable hubs, however experiences basic issues, for example, constrained location space, power utilization, handover inactivity, parcel misfortune and so on. To overcome these problems, Internet Engineering Task Force proposed Protocol version 6 for Mobile Internet as the first Internet Protocol version 6-based convey ability the board show. Protocol version 6 for Mobile Internet decreases these issues up to a degree yet at the same time these issues are significant difficulties for versatility the board plans. In 2008,
Internet Engineering Task Force has put forth PMIPv6 as first system based portability the executives plot. In it, organize substances are liable for versatility the executives for portable hub, in this way decreasing force utilization issue. What's more, it diminishes handover dormancy; bundle misfortune and correspondence delay, yet at the same time experiences security problems. Later on, scholars put forth Optimized and Unassailable Scheme of Authentication in Proxy Mobile Internet Protocol version 6, regarded as the finest between plot considered for examination.

\section{References}

[1] Cisco Systems Inc., "Cisco Visual Networking Index: Global Mobile Data Traffic Forecast Update, 2016-2021", March 2017.

[2] Han-Chieh Chao, Wei-Ming Chen and Yen-Ming Chu, "A Low Latency Handoff Algorithm for Voice over IP Traffics in the Next Generation Packet-Based Cellular Networks: Cellular Mobile IPv6", Wireless Personal Communications, vol. 23, issue 3, pp. 353-378, December 2002.

[3] Praveen Muley, Geng Liang and Hans Liu, "Network based Bonding solution for Hybrid Access", Internet Draft, November 2016. 
[4] J. Korhonen, J. Soininen, B. Patil, T. Savolainen, G. Bajko and K. Iisakkila, "IPv6 in 3rd Generation Partnership Project (3GPP) Evolved Packet System (EPS)", RFC 6459, January 2012.

[5] Z. Becvar, P. MacH, and B. Simak, "Improvement of handover prediction in mobile WiMAX by using two thresholds," Computer Networks, vol. 55, no. 16, pp. 3759-3773, November 2011.

[6] Riky Subrata and Albert Y. Zomaya, "Dynamic Location Management for Mobile Computing", Telecommunication Systems, Vol. 22, issue 1-4, pp. 169-187, January 2003.

[7] Javier Carmona-Murillo José-Luis González-Sánchez David Cortés-Polo Francisco-Javier and Rodríguez-Pérez, "QoS in Next Generation Mobile Networks: An Analytical Study", Resource Management in Mobile Computing Environments, vol. 3, pp. 25-41, 2014.

[8] Hyun-Suk Chai, Jongpil Jeon and Chul-Hee Cho, "Security analysis of fast inter-LMA domain handover scheme in proxy mobile IPv6 networks", Pervasive and Mobile Computing, vol. 39, pp. 100-116, August 2017.

[9] Arun Kumar Tripathi, J. S. Lather and R. Radhakrishnan, "Secure and Optimized Authentication Scheme in Proxy Mobile IPv6 (SOAS-PMIPv6) to reduce Handover Latency", I. J. Computer Network and Information Security, vol. 9, issue 10, pp. 1-12, October 2017.
[10] Arun Kumar Tripathi, R. Radhakrishnan and J. S. Lather, "One Time Authentication Scheme (OTAS) for Reducing Handover Latency in Proxy Mobile IPv6", International Conference on Communication and Computing (ICC2014), pp. 324-331, 12-14 June 2014.

[11] Arun Kumar Tripathi, and Surendra Kumar Tripathi, "A Quality Analysis of Secured Handover Management Schemes for Mobile IPv6 Enabled Network", $20183^{\text {rd }}$ International Innovative Application of Computational Intelligence on power, Energy and Controls with their impact on Humanity (CIPECH), 2018.

[12] D. Johnson, C. Perkins and J. Arkko, "Mobility support in IPv6", RFC 3775, June 2004.

[13] Mo Lin-Li, "Research on Mobile IPV6 Technology and Handover Performance Optimization", Advances in Intelligent Systems and Computing book series (AISC, volume 181).

[14] Yang Li-shen, Li Jing, "Research and Technique of IPv6 Flow Control Based on Net filter framework and HTB", Proceedings of the Third International Symposium on Electronic Commerce and Security Workshops (ISECS '10).

[15] LIU Zheng, CAI Ming, "A Hierarchical Mobile IPv6 Optimization Scheme", Computer Engineering \& Science, 2010 .

\section{Biography}

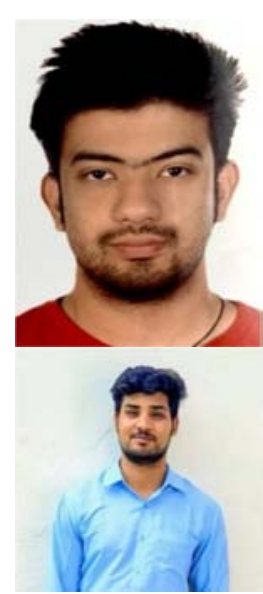

Harshit Kargeti was born in Aligarh, India in 1994. He received his B. Tech degree in Information \& Technology from ABES Institute of Technology in 2017. He founded his Startup named Fittrage LLP in 2016. He joined Isoftnic Pvt Ltd. in 2017 as a software engineer. In 2019 he Joined Biz2Credit as a software engineer and in 2020 He joined Wayforward wellness as a senior software engineer. Currently he is a software Developer at Algoworks Noida. His research interest includes Big data analytics, AI, smart voice recognition, etc

Arpit Bhushan Sharma was born in Aligarh, India, in 1997. He got his B. Tech from Department of Electrical \& Electronics Engineering from KIET Group of Institutions, Ghaziabad, India in year 2020. He is currently working as a Engineering Patent Analyst at Lakshmikumaran \& Sridharan Attorney, New Delhi and a Part-time AI developer in 10xAI learning Noida. He got first prize from Institute of Scholars $(\mathrm{InSc})$ for his final year project and become the lifetime member of InSc. He is also a Student Member in Institution of Electrical and Electronics Engineers (IEEE) USA and Serving as a Beta Microsoft Student Learn Ambassador. His research interest includes Artificial Intelligence, Machine Learning, Deep Learning, computational Intelligence and NLP etc. 